\title{
Antischistosomal activity of a calcium channel antagonist on schistosomula and adult Schistosoma mansoni worms
}

\author{
Vanessa Silva-Moraes ${ }^{1,3}$, Flávia Fernanda Bubula Couto ${ }^{1}$, Marah Mileib Vasconcelos ${ }^{2}$, \\ Neusa Araújo1, Paulo Marcos Zech Coelho' ${ }^{1 /}$, Naftale Katz ${ }^{1}$, Rafaella Fortini Queiroz Grenfell1,2
}

\author{
'Laboratório de Esquistossomose, Centro de Pesquisas René Rachou-Fiocruz, Belo Horizonte, MG, Brasil \\ ²Escola de Farmácia, Universidade de Itaúna, Itaúna, MG, Brasil ³ Universidade Federal de São João Del Rey, Divinópolis, MG, Brasil
}

\begin{abstract}
Current schistosomiasis control strategies are largely based on chemotherapeutic agents and a limited number of drugs are available today. Praziquantel (PZQ) is the only drug currently used in schistosomiasis control programs. Unfortunately, this drug shows poor efficacy in patients during the earliest infection phases. The effects of PZQ appear to operate on the voltage-operated $\mathrm{Ca}^{2+}$ channels, which are located on the external Schistosoma mansoni membrane. Because some $\mathrm{Ca}^{2+}$ channels have dihydropyridine drug class (a class that includes nifedipine) sensitivity, an in vitro analysis using a calcium channel antagonist (clinically used for cardiovascular hypertension) was performed to determine the antischistosomal effects of nifedipine on schistosomula and adult worm cultures. Nifedipine demonstrated antischistosomal activity against schistosomula and significantly reduced viability at all of the concentrations used alone or in combination with PZQ. In contrast, PZQ did not show significant efficacy when used alone. Adult worms were also affected by nifedipine after a $24 \mathrm{~h}$ incubation and exhibited impaired motility, several lesions on the tegument and intense contractility. These data support the idea of $\mathrm{Ca}^{2+}$ channels subunits as drug targets and favour alternative therapeutic schemes when drug resistance has been reported. In this paper, strong arguments encouraging drug research are presented, with a focus on exploring schistosomal $\mathrm{Ca}^{2+}$ channels.
\end{abstract}

Key words: Schistosoma mansoni - antischistosomal drugs - calcium channels antagonists - L-type calcium channels - nifedipine

Schistosomiasis remains a major health problem in many tropical areas. Although it causes more than 280,000 deaths annually (Chitsulo et al. 2004), current disease control strategies are primarily based on improving water supplies, basic sanitation, snail control and chemotherapeutic agents (Fenwick \& Webster 2006). Among these strategies, in endemic areas, chemotherapy is extremely important for preventing disease mobility and controlling transmission. However, there are limited drug options available and praziquantel (PZQ) has been classified as the drug of choice by the World Health Organization. Although PZQ shows significant efficacy in most cases and is a low-cost treatment (Fenwick et al. 2003, Coura \& Amaral 2004), drug resistance has been increasingly reported (Botros \& Bennett 2007, Caffrey 2007, Beckmann et al. 2012). Additionally, PZQ is ineffective at killing the immature worms that are present in recently acquired infections, which leads to reduced cure rates (Gönnert \& Andrews 1977, Pica-Mattoccia et al. 2007, Doenhoff et al. 2008, El-Lakkany et al. 2011) and reinforces the need to develop new alternative drugs.

Schistosoma mansoni undergo a strong calcium $\left(\mathrm{Ca}^{2+}\right)$ influx within seconds of PZQ exposure; this influx is followed by intense and sustained muscular contraction, disruption of the parasite tegument and death (Pax et al.

doi: 10.1590/0074-0276108052013011

Financial support: CNPq, FIOCRUZ, CAPES, UIT

+ Corresponding author: coelhopm@cpqrr.fiocruz.br

Received 29 November 2012

Accepted 18 December 2012
1978). In addition, the cytosolic $\mathrm{Ca}^{2+}$ concentrations are controlled by channels located on the tegument; these channels are known to be essential because the parasite lacks overall cell physiology (Noël et al. 2001). Although the PZQ receptor has not yet been definitively identified, several studies have demonstrated that voltage-operated $\mathrm{Ca}^{2+}$ channels (VOCC) are prime candidate targets for chemotherapy (Redman et al. 1996, Greenberg 2005a), as they have a critical role in regulating the levels of intracellular $\mathrm{Ca}^{2+}$ and are essential for a variety of parasite cellular events, including contraction, gene expression and neurotransmitter release (Salvador-Recatalà et al. 2008).

VOCCs are heteromultimeric membrane protein complexes that are composed of principal $\alpha_{1}$ subunit and auxiliary $\alpha_{2} \beta$ and $\delta$ subunits. The $\alpha_{1}$ subunit comprises four linked homologous domains with six transmembrane segments pore-forming the $\mathrm{Ca}^{2+}$ channel (Catterall 2000).

Several studies have sought to identify new alternative drugs to treat schistosomiasis (Fenwick \& Webster 2006, Caffrey 2007, Araújo et al. 2008a, b, El-Lakkany et al. 2011) and have shown promising results, although the individual mechanisms of action have not yet been reported. In this study, we utilised the well-known mechanism of nifedipine on L-type $\mathrm{Ca}^{2+}$ channels to evaluate the antischistosomal effect of this drug alone and combined with PZQ on schistosomula and adult worm cultures.

\section{MATERIALS AND METHODS}

In vitro schistosomula experiments - Cercariae (LE strain) were obtained from the Laboratory of Malacology at the René Rachou Research Centre/Oswaldo Cruz Foundation (Fiocruz) and were mechanically transformed to schistosomula using the modified technique 
of Ramalho-Pinto et al. (1974). Briefly, cercariae were placed in conical tubes and left in an ice bath for $30 \mathrm{~min}$ before centrifugation (Eppendorf Centrifuge 5820R) at $200 \mathrm{~g}$ for $3 \mathrm{~min}$ at $4^{\circ} \mathrm{C}$. The pellet was resuspended in frozen RPMI-1640 culture medium (Sigma Chemical Co, USA). The cercarial tails were broken through vortexing (Scientific Industries Genie-2) at maximum speed for $2 \mathrm{~min}$. Later, the tails were removed from the medium through repeated washing steps with $0.9 \%$ saline solution followed by centrifugation at $200 \mathrm{~g}$ for $1 \mathrm{~min}$ and the culture was checked for high viability $(\leq 2 \%$ dead or damaged larvae) prior to use for screening. Forty $( \pm 5)$ schistosomula per well were then transferred to six-well plates containing $3 \mathrm{~mL}$ of medium supplemented with $5 \%$ foetal bovine serum (FBS) (Gibco Limited, Scotland, UK) and $100 \mu \mathrm{g} / \mathrm{mL}$ of penicillin/streptomycin antibiotics (Sigma Chemical Co. P4333, USA) and were incubated in $5 \% \mathrm{CO}$ incubator at $37^{\circ} \mathrm{C}$. Nifedipine alone or combined with PZQ was added to the culture for different incubation times after solubilisation in $20 \mu \mathrm{L}$ of methanol. For the first experiment, drugs were added after $2 \mathrm{~h}$ following schistosomula adaptation to the culture medium. For the second experiment, the drug was added after seven days of incubation. The following concentrations of nifedipine/PZQ were used: 0.60/1.20, 1.20/2.0 and 2.40/4.0 $\mu \mathrm{g} / \mathrm{mL}$, respectively. After $2 \mathrm{~h}$ of drug introduction, the parasites were monitored with a stereoscopic microscope (Zeiss Stemi DV4) and the mortality rate, motility and tegument damage were evaluated. The control wells contained schistosomula in medium without drugs, both with and without methanol. Each drug concentration was evaluated in triplicate ( 3 independent experiments).

In vitro adult worm experiments - Mice infected with $100( \pm 10)$ cercariae (LE strain) subcutaneously were submitted to hepatic portal system perfusion 45 days after infection (Smithers \& Terry 1965); culture medium RPMI-1640 with $0.2 \%$ heparin (Blausiegel, Brazil) was used as the perfusate. Four viable adult worms pairs were distributed in each well of the six-well plates containing $3 \mathrm{~mL}$ of medium supplemented with 5\% FBS (Gibco Limited, Scotland, UK) and $100 \mu \mathrm{g} /$ $\mathrm{mL}$ penicillin/streptomycin antibiotics (Sigma Chemical Co, USA). Afterwards, nifedipine, alone or combined with PZQ and solubilised in methanol, was added to the wells. The concentrations of nifedipine and PZQ used were $0.25 / 1.20,0.50 / 2.0$ and $1.0 / 4.0 \mu \mathrm{g} / \mathrm{mL}$, respectively. The plates were incubated in $5 \% \mathrm{CO}_{2}$ at $37^{\circ} \mathrm{C}$ for $24 \mathrm{~h}$. The worms were washed five times with $2 \mathrm{~mL}$ medium to remove the drug and then incubated in drug-free me$\operatorname{dium}\left(5 \% \mathrm{CO}_{2}\right)$ at $37^{\circ} \mathrm{C}$ for seven days. The worms were analysed under an optical microscope daily to evaluate the mortality rate, motility and damage to the tegument. Each drug concentration was evaluated in triplicate (3 independent experiments).

Statistical analysis - The data were analysed with the Minitab software (Minitab Inc, USA) using the Kolmogorov-Smirnov normality test. Normally distributed data were analysed with Student's $t$ test and non-normally distributed data were analysed with the Mann-Whitney $U$ test (the significance level was set at $\mathrm{p}$ value $\leq 0.05$ ).

Ethics - The use of animals was approved by the Ethical Commission in the Use of Animals, Fiocruz, Brazil (L-0023/08).

\section{RESULTS}

In vitro schistosomula experiments - Varying concentrations of nifedipine were used either alone or combined with PZQ and the schistosomula mortality rate was evaluated in each group after drug introduction in the two-hour and seven-day cultures. Nifedipine strongly reduced schistosomula viability after both $2 \mathrm{~h}$ and seven days in all the evaluated concentrations when used alone $(\mathrm{p}=0.03)$ or in combination with PZQ ( $p$ $=0.02)$, as shown in Fig. 1. PZQ did not significantly reduce viability at the concentrations used, although a microscopic analysis indicated that the muscles of schistosomula contracted when the highest concentration of PZQ was added to the culture. Tegumental damage was also observed following the nifedipine treatment.

In vitro adult worm experiments - Nifedipine, either alone or in combination with PZQ, was added to cultures containing four pairs of adult worms; microscopy observations from this experiment are shown in the Table. Alterations in worm behaviour were observed following the addition of nifedipine to the culture. Decreased or absent motility was observed in all the cultures in which the worms had contracted muscles and several lesions
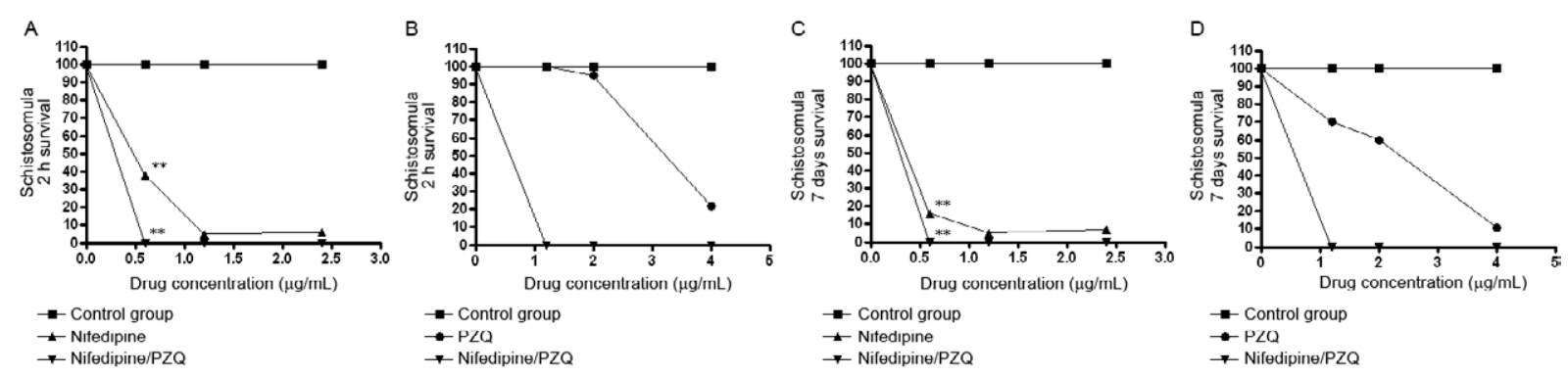

Fig. 1: percentage of schistosomula survival after $2 \mathrm{~h}$ incubation with nifedipine combined or not with praziquantel (PZQ). Schistosomula incubation was done for recently transformed schistosomula (A, B) and for seven days cultured schistosomula (C, D). Statistical differences were determined by Student's $t$ test or Mann-Whitney $U$ test. Differences are represented by asterisks $(\mathrm{p} \leq 0.05)$. 
were noticed on the tegument of male and female parasites. Nifedipine also altered egg deposition, as no eggs were detected in any culture. In contrast, PZQ, when used alone, was not capable of reducing egg deposition and eggs of all stages were founded in the cultures. When nifedipine was combined with PZQ, low motility was observed and no viable eggs were observed in the culture. Tegumental injures were more apparent following nifedipine exposure compared to PZQ, particularly for the highest concentration used, which induced significant disruption of the tegument. Following the removal of the drugs at the end of seven days of incubation, the worms were still contracted and exhibited no motility. They also presented no tegument colour and were considered dead. The microscopy visualisation is shown in Fig. 2. In contrast, eggs from multiple different stages were found in the control group, along with intense worm motility and no alterations in worm morphology or tegument damage.

\section{DISCUSSION}

Nifedipine is a potent L-type VOCC antagonist that is commonly used to treat hypertension. After gastrointestinal absorption, nifedipine easily attaches to the VOCC located on the endothelium of blood vessels. Once VOCC is blocked, $\mathrm{Ca}^{2+}$ input is also blocked and the endothelium cells undergo intense relaxation, thereby reducing circulating blood pressure (Triggle 2007, Varon 2008).

The L-type $\alpha_{1}$ VOCC subunit $\left(S m \mathrm{Ca}_{\mathrm{v}} 1\right)$ is one of the two VOCC subunits in the $S$. mansoni genome. $S m \mathrm{Ca}_{1} 1$ and vertebrate homologues have approximately $50-55 \%$ structural similarity, such as the four homologous domains and a pore composed of the area between the S5 and S6 transmembrane regions in each domain (Kohn et al. 2001a). Other authors have shown that antagonist drugs, such as nifedipine, modulate the channel through allosteric binding at different sites in the centre $\alpha_{1}$ pore region S6 in domains III and IV and the S5-S6 linker in domain III (Hockerman et al. 1997, Striessnig et al. 1998).
Based on the antagonist mechanism of nifedipine activity and on the similarity between S. mansoni VOCC and vertebrates VOCC, we evaluated the effect of this drug on schistosomula and adult worm cultures to provide additional data for new therapeutic strategies for schistosomiasis mansoni treatment. These experiments showed promising initial results for nifedipine, which displayed a significant antischistosomal effect even on the initial life cycle stages of the parasite, such as schistosomula. Amlodipine and diltiazem were also included in preliminary assays and both demonstrated in vitro antischistosomal activity that was similar to nifedipine (data not shown), although nifedipine presented the strongest effect and was exclusively used for further analysis.

The antischistosomal effects of nifedipine on schistosomula deserve attention. When nifedipine was added

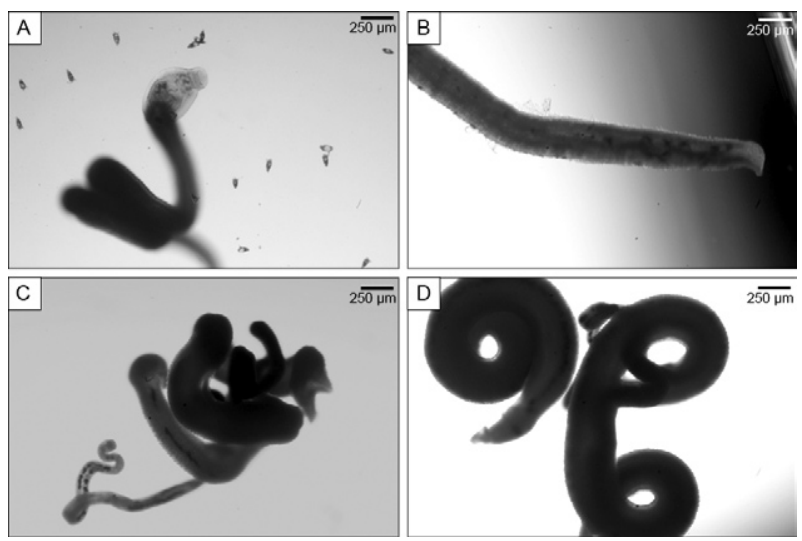

Fig. 2: microscopy visualisation of adult worms after $24 \mathrm{~h}$ incubation with nifedipine. A: control group showing no alteration on morphology or tegument and the presence of eggs; B: adult worm after nifedipine were added showing damage on the tegument; $\mathrm{C}, \mathrm{D}$ : adult worms with contorted muscle and absence of eggs after incubation with nifedipine.

TABLE

Microscopy analysis of adult worms cultured for $24 \mathrm{~h}$ with nifedipine alone or combined with $2.0 \mu \mathrm{g} / \mathrm{mL}$ of praziquantel (PZQ)

\begin{tabular}{|c|c|c|c|c|}
\hline & $\begin{array}{l}\text { Concentration } \\
\qquad(\mu \mathrm{g} / \mathrm{mL})\end{array}$ & $\begin{array}{l}\text { Motility } \\
\text { intensity }\end{array}$ & $\begin{array}{c}\text { Morphology } \\
\text { aspect }\end{array}$ & $\begin{array}{l}\text { Viable } \\
\text { eggs }\end{array}$ \\
\hline Control group & 0 & Hyperactivity & No alteration & All stages \\
\hline Nifedipine & $\begin{array}{c}0.25 \\
0.50 \\
1.0\end{array}$ & $\begin{array}{l}\text { Low } \\
\text { No motility } \\
\text { No motility }\end{array}$ & $\begin{array}{l}\text { Contraction } \\
\text { Contraction } \\
\text { Contraction }\end{array}$ & $\begin{array}{l}\text { No eggs } \\
\text { No eggs } \\
\text { No eggs }\end{array}$ \\
\hline PZQ & $\begin{array}{l}1.2 \\
2.0 \\
4.0\end{array}$ & $\begin{array}{l}\text { Hyperactivity } \\
\text { Hyperactivity } \\
\text { Low }\end{array}$ & $\begin{array}{l}\text { No alteration } \\
\text { Contraction } \\
\text { Contraction }\end{array}$ & $\begin{array}{l}\text { All stages } \\
\text { All stages } \\
\text { All stages }\end{array}$ \\
\hline Nifedipine/PZQ & $\begin{array}{c}0.25 / 1.20 \\
0.50 / 2.0 \\
1.0 / 4.0\end{array}$ & $\begin{array}{c}\text { Low } \\
\text { Low } \\
\text { No motility }\end{array}$ & $\begin{array}{l}\text { Contraction } \\
\text { Contraction } \\
\text { Contraction }\end{array}$ & $\begin{array}{l}\text { No eggs } \\
\text { No eggs } \\
\text { No eggs }\end{array}$ \\
\hline
\end{tabular}


to the cultures, schistosomula showed significantly reduced viability at all the concentration levels used after both $2 \mathrm{~h}$ and seven days of culture and these observations were noticed for the cultures in which nifedipine was used either alone or combined with PZQ. Parasites treated in this manner showed impaired viability and exhibited tegument injuries. Although PZQ has reduced effectiveness on immature worms and schistosomula (Gönnert \& Andrews 1977, Doenhoff et al. 2008, ElLakkany et al. 2011), our data showed that PZQ did not interfere with the effects of nifedipine when both drugs were combined, which suggested that these drugs do not compete for access to the same parasite VOCC site. These data corroborate other findings that the $\beta$ subunit of parasite VOCC is a proposed PZQ target (Kohn et al. 2003, Greenberg 2005a). Jointly, based on the observations of Fetterer et al. (1980), the blocking of $\mathrm{Ca}^{2+}$ conductance through VOCC strongly interferes with cellular ionic homeostasis, which may explain the lack of viability and the injuries on the parasite tegument after nifedipine treatment.

Similar results were observed when nifedipine was added to adult worm cultures. Impaired or absent motility was observed in these adult worms (male and female) together with several other tegumental injuries. Spasms were also observed when the highest concentrations of nifedipine were added to the culture. Collectively, the entire parasite body was contracted after drug exposure, which may have been caused by the depleted intracellular $\mathrm{Ca}^{2+}$ stores, which occurred when the VOCC was blocked and eliminated the extracellular $\mathrm{Ca}^{2+}$ influx (Pax et al. 1978). Other researchers have described this response after only 10 min without $\mathrm{Ca}^{2+}$ (reviewed by Greenberg 2005b). Our data not only supported this observation, but also showed that the same response was maintained after $24 \mathrm{~h}$ of drug exposure.

No eggs were produced by female adult worms after the nifedipine treatment (used alone or in combination with PZQ), which suggested that the drug significantly impaired parasite fecundity. These observations were also made by others when verapamil, another $\mathrm{Ca}^{2+}$ channel antagonist, was used (Walter \& Kuris 2003). Bonn (2004) also emphasised that the interruption of the egg production is extremely relevant because eggs are responsible for most of the lesions witnessed in infected patients. PZQ alone, however, did not show any effect on egg deposition. Additionally, the tegumental injuries were more expressive following exposure to nifedipine compared to PZQ alone.

PZQ is considered a $\mathrm{Ca}^{2+}$ channel agonist that may disrupt the interaction of the $\alpha_{1}$ and $\beta$ subunits, which would allow more $\mathrm{Ca}^{2+}$ channels to open and lead to the disruption of normal $\mathrm{Ca}^{2+}$ homeostasis (Kohn et al. 2001b). Despite the agonist effect of PZQ on $\mathrm{Ca}^{2+}$ channels, co-treatment with PZQ and nifedipine, a $\mathrm{Ca}^{2+}$ channel antagonist, did not block the effects of nifedipine, as nifedipine presented similar antischistosomal activity alone or in combination with PZQ.

The possibility that VOCC could be the target of antischistosomal drug activity has been the focus of many studies using distinct assays to characterise the struc- ture, function and pharmacological sensitivity of the channels present in the parasite (Day et al. 1993, Bonn 2004, Mendonça-Silva et al. 2006, Pica-Mattoccia et al. 2007). In summary, our work reinforced the importance of the VOCC in the different life cycles stages of S. mansoni and raised the possibility of using drugs that interfere directly with $\mathrm{Ca}^{2+}$ homeostasis as a schistosomiasis treatment, potentially helping to solve the emerging PZQ resistance problem. We demonstrated a new alternative drug with significant in vitro antischistosomal effects against schistosomula and adult $S$. mansoni worms. Preliminary in vivo studies on murine model have revealed that the combination of nifedipine and PZQ did not show significant efficacy. Together, no toxicity was observed after oral nifedipine administration. We recommend further analysis to better understand the in vivo efficacy of this new potential treatment for schistosomiasis.

\section{REFERENCES}

Araújo N, de Mattos ACA, Coelho PMZ, Katz N 2008a. Association of oxamniquine praziquantel and clonazepam in experimental schistosomiasis mansoni. Mem Inst Oswaldo Cruz 103: 781-785.

Araújo N, de Mattos ACA, Sarvel AK, Coelho PMZ, Katz N 2008b. Oxamniquine, praziquantel and lovastatin association in the experimental schistosomiasis mansoni. Mem Inst Oswaldo Cruz 103: $450-454$.

Beckmann S, Leutner S, Gouignard N, Dissous C, Grevelding CG 2012. Protein kinases as potential targets for novel anti-schistosomal strategies. Curr Pharm Des 18: 3579-3594.

Bonn D 2004. Schistosomiasis: a new target for calcium channel blockers? Lancet Infect Dis 4: 190.

Botros S, Bennett JL 2007. Praziquantel resistance. Expert Opin Drug Discov 2: 535-540.

Caffrey CR 2007. Chemotherapy of schistosomiasis: present and future. Curr Opin Chem Biol 11: 433-439.

Catterall WA 2000. Structure and regulation of voltage-gated $\mathrm{Ca}^{2+}$ channels. Annu Rev Cell Dev Biol 16: 521-555.

Chitsulo L, Loverde P, Engels D 2004. Schistosomiasis. Nat Rev Microbiol 2: 12-13.

Coura JR, Amaral RS 2004. Epidemiological and control aspects of schistosomiasis in Brazilian endemic areas. Mem Inst Oswaldo Cruz 99 (Suppl. I): 13-19.

Day TA, Orr N, Bennett JL, Pax RA 1993. Voltage-gated currents in muscle cells of Schistosoma mansoni. Parasitology 106: 471-477.

Doenhoff MJ, Cioli D, Utzinger J 2008. Praziquantel: mechanisms of action, resistance and new derivatives for schistosomiasis. Curr Opin Infect Dis 21: 659-667.

El-Lakkany NM, el-Din SHS, Sabra ANAA, Hammam OA 2011. Pharmacodynamics of mefloquine and praziquantel combination therapy in mice harbouring juvenile and adult Schistosoma mansoni. Mem Inst Oswaldo Cruz 106: 814-822.

Fenwick A, Savioli L, Engels D, Robert Bergquist NR, Todd MH 2003. Drugs for the control of parasitic diseases: current status and development in schistosomiasis. Trends Parasitol 19: 509-515.

Fenwick A, Webster JP 2006. Schistosomiasis: challenges for control, treatment and drug resistance. Curr Opin Infect Dis 19: 577-582.

Fetterer RH, Pax RA, Bennett JL 1980. Praziquantel, potassium and 2,4-dinitrophenol: analysis of their action on the musculature of Schistosoma mansoni. Eur J Pharmacol 64: 31-38. 
Gönnert R, Andrews P 1977. Praziquantel, a new board-spectrum antischistosomal agent. Z Parasitenkd 52: 129-150.

Greenberg RM 2005a. Are $\mathrm{Ca}^{2+}$ channels targets of praziquantel action? Int J Parasitol 35: 1-9.

Greenberg RM 2005b. $\mathrm{Ca}^{2+}$ signalling, voltage-gated $\mathrm{Ca}^{2+}$ channels and praziquantel in flatworm neuromusculature. Parasitology 131: $97-108$.

Hockerman GH, Peterson BZ, Johnson BD, Catterall WA 1997. Molecular determinants of drug binding and action on L-type calcium channel. Annu Rev Pharmacol Toxicol 37: 361-396.

Kohn AB, Anderson PAV, Roberts-Misterly JM, Greenberg RM 2001b. Schistosome calcium channel $\beta$ subunits: unusual modulatory effects and potential role in the action of the antischistosomal drug praziquantel. J Biol Chem 276: 36873-36876.

Kohn AB, Lea JM, Roberts-Misterly JM, Anderson PAV, Greenberg RM 2001a. Structure of three high voltage-activated $\mathrm{Ca}^{2+}$ channel $\alpha_{1}$ subunits from Schistosoma mansoni. Parasitology 123: 489-497.

Kohn AB, Roberts-Misterly JM, Anderson PAV, Khan N, Greenberg RM 2003. Specific sites in the beta interaction domain of a schistosome $\mathrm{Ca}^{2+}$ channel $\beta$ subunit are key to its role in sensitivity to the antischistosomal drug praziquantel. Parasitology 127: 349-356.

Mendonça-Silva DL, Novozhilova E, Cobbett PJ, Silva CL, Noël F, Totten MI, Maule AG, Day TA 2006. Role of calcium influx through voltage-operated calcium channels and of calcium mobilization in the physiology of Schistosoma mansoni muscle contractions. Parasitology 133: 67-74.

Noël F, Cunha VMN, Silva CLM, Mendonça-Silva DL 2001. Control of calcium homeostasis in Schistosoma mansoni. Mem Inst Oswaldo Cruz 96 (Suppl.): 85-88.
Pax R, Bennett JL, Fetterer R 1978. A benzodiazepine derivative and praziquantel: effects on musculature of Schistosoma mansoni and Schistosoma japonicum. Naunyn Schmiedebergs Arch Pharmacol 304: 309-315.

Pica-Mattoccia L, Ruppel A, Xia CM, Cioli D 2007. Praziquantel and the benzodiazepine Ro 11-3128 do not compete for the same binding sites in schistosomes. Parasitology 135: 47-54.

Ramalho-Pinto FJ, Gazzinelli G, Howells RE, Mota-Santos TA, Figueiredo EA, Pellegrino J 1974. Schistosoma mansoni: defined system for stepwise transformation of cercaria to schistosomula in vitro. Exp Parasitol 36: 360-372.

Redman CA, Robertson A, Fallon PG 1996. Praziquantel: an urgent and exciting challenge. Parasitol Today 12: 14-20.

Salvador-Recatalà V, Schneider T, Greenberg RM 2008. Atypical properties of a conventional calcium channel beta subunit from the platyhelminth Schistosoma mansoni. BMC Physiol 8: 6.

Smithers SR, Terry RJ 1965. The infection of laboratory hosts with cercariae of Schistosoma mansoni and the recovery of adult worms. Parasitology 55: 695-700.

Striessnig J, Grabner M, Mitterdorfer J, Hering S, Sinnegger MJ, Glossmann H 1998. Structural basis of drug binding to $\mathrm{L} \mathrm{Ca}^{2+}$ channels. Trends Pharmacol Sci 19: 108-115.

Triggle DJ 2007. Calcium channel antagonists: clinical uses-past, present and future. Biochem Pharmacol 74: 1-9.

Varon J 2008. Treatment of acute severe hypertension: current and newer agents. Drugs 68: 283-297.

Walter M, Kuris A 2003. Methods for the inhibition of egg production in trematodes. USA patent 6,514,963. 2003; B2. 